\title{
Fatal attraction
}

DOI:

10.1038/nrmicro1687
It is known that infection of rats with the protozoan parasite Toxoplasma gondii alters the rat's innate aversion to cat urine, and can even result in an attraction to cat pheromones. However, it was unknown whether this is an adaptive, behavioural manipulation by T. gondii, or whether the parasite disrupts innate and learned fear non-specifically. Now, in a study published in Proceedings of the National Academy of Sciences USA, Vyas et al. have begun to resolve this question.

T. gondii reproduces sexually using two hosts. Excreted oocysts eaten by grazing rodents form cysts in the rodent's central nervous system and persist latently in the brain for life. By blocking the innate aversion of rats for cat urine, T. gondii increases the likelihood of a cat predating a rat, which increases the chances of the parasite re-entering the feline intestine, where it can reproduce sexually, completing its lifecycle. The 'behavioural manipulation' hypothesis states that a parasite will specifically manipulate host behaviour to increase its own transmission efficiency. The question Vyas et al. wanted to answer was whether this manipulation specifically affects the rodent's innate aversion to cat urine, and so agrees with the hypothesis, or whether infection results in a general compromise of a range of defensive behaviours (a non-specific response), which conflicts with the hypothesis. A third possibility is that the behavioural change is merely a side effect of sickness.

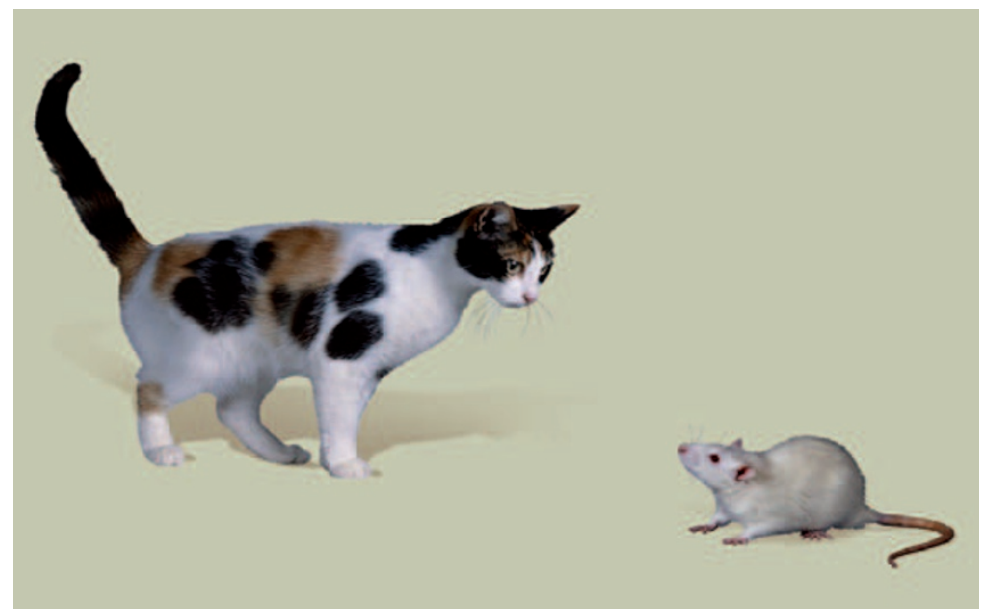

Using rats and mice, the study found that $T$. gondii infection invoked a highly specific response. As olfaction and growth rate and energetically expensive behaviours were all unaffected, the 'side effect of sickness' hypothesis was ruled out. The neural circuits used in innate fear, learned fear and anxiety overlap. However, the authors showed that T. gondii only affects the part of the defensive reaction that is important for its own transmission - the innate aversion to cat pheromones. Infected animals showed no decrease in their aversion to dog urine, a rodent predator that is not suitable for T. gondii reproduction. In addition, the parasite actually causes rats to be attracted to cat urine, ruling against a passive behavioural effect.

Taken together, these findings show that latent $T$. gondii infection specifically alters the behaviour of the rodent host for its own selective benefit, rather than decreasing learned fear or anxiety-like behaviour. The mechanisms involved remain unknown and require further investigation.

Elinor Faulkner

ORIGINAL RESEARCH PAPER Vyas, A., Kim, SK., Giacomini, N., Boothroyd, J. C. \& Sapolsky, R. M. Behavioral changes induced by Toxoplasma infection of rodents are highly specific to aversion of cat odours. Proc. Natl Acad. Sci. USA 104, 64426447 (2007) 